\title{
ON FILTER EXPANSION OF TOPOLOGIES
}

\author{
M.E. ABD EL-MONSEF, A.M. KOZAE AND A.A.ABO KHAORA
}

\begin{abstract}
The lower separation axioms $T_{i},(i \in\{0,1,2\})$ are obviously preserved under topology expansions. This fact is not generally valid for higher separation axioms as well as for recent sorts of separation such as $T_{R}, R_{0}, R_{1}$ and semi- $R_{i},(i \in$ $\{0,1\})$. The purpose of the present work is to investigate preservation of these recent separation properties under filter expansion of topologies. Also, we study the effect of filter expansions on the concept s-essentially $T_{i}$ - spaces, $(i \in\{0,1\})$
\end{abstract}

\section{Introduction}

For any subset $A$ of a topological space $(X, \tau)$ we denote the closure (resp. the interior) of $A$ with respect to $\tau$ by $\operatorname{cl}_{\tau} A$ (resp. $\operatorname{int}_{\tau} A$ ). A subset $\mathrm{A}$ is said to be semiopen [151 (resp. $\alpha$-open [17]) if $A \subset \mathrm{cl}$ (int $A$ ) (resp. $A \subset \operatorname{int~(cl~(int~} A$ ). $A$ is semiclosed [4] if $(X-A)$ is semiopen. We denote the family of all semiopen (resp. semi-closed, $\alpha$-open) sets in $(X, \tau)$ by $S O(X, \tau)$ (resp. $\left.S C(x, \tau), \tau^{\alpha}\right)$ ). Njasted [17] has shown that $\tau^{\alpha}$ is a. topology on $X$ satisfying $\tau \subset \tau^{\alpha}$. The semi closure of $A$ [4] (denoted by s-cl $A$ ) is the intersection of all semiclosed sets containing $A$. $A$ space $X$ is submaximal [3] iff all dense subsets are open and is resolvable [11], if there is a subset $D$ of $X$ such that $D$ and $X-D$ are both dense in $X$ and it is irresolvable if it is not resolvable.

Definition 1.1. [1] Let $(X, \tau)$ be a space and $\mathcal{F}$ be a filter on $X$, then the topology $\tau(\mathcal{F})=\{U \cap F: U \in \tau, F \in \mathcal{F}\}$ is called a filter expansion of $\tau$. We remark that $d_{\tau(\mathcal{F})} A \subset d_{\tau} A$, for any $A \subset X$, where $d_{\tau(\mathcal{F})}, d_{\tau}$ denote the derived operators relative to $\tau(\mathcal{F})$ and $\tau$, respectively

Definition 1.2. [18] A space $(X, \tau)$ is $T_{R}$ iff for every $x \in X$, either $d\{x\}$ is empty or $d\{x\}$ contains a non-empty closed set.

Received August 30, 1992.

1991 AMS Subject Classification : 54 A 10, 54 C10, 54 D 20.

Key words and phrases: Semiopen and $\alpha$-open sets, filter expansion of topologies, semi- $T_{0}$-identification space, $T_{R}, R_{0}, R_{1}$ and semi- $R_{i}, i \in\{0,1\}$. 
Definition 1.3. [18] A space $(X, \tau)$ is $C_{R}$ iff for every $x \in X, d\{x\}$ does not contain a non-empty closed set.

Definition 1.4. [6] In a space $(X, \tau)$, let $x \in X$. Then the kernal of $x$, $\{\hat{x}\}=$ $\cap\{O / x \in O, O \in \tau\}$ and the covering of $x,\langle x\rangle=\operatorname{cl}\{x\} \cap\{\hat{x}\}$

Definition 1.5. [13] In a space $(X, \tau)$, we will call superderived of $x, x \in X$, the set $D\{x\}=\operatorname{cl}\{x\}-\langle x\rangle$, that is the union of the closed sets which are contained in $d\{x\}$.

Definition 1.6. [5] A space $(X, \tau)$ is $R_{0}$ iff for orbitrary $x, y \in X$, either cl $\{x\}=\operatorname{cl}\{y\}$ or $\operatorname{cl}\{x\} \cap \operatorname{cl}\{y\}=\emptyset$

Definition 1.7. [5] A space $(X, \tau)$ is $R_{1}$ iff for each pair $x, y \in X$ such that $\operatorname{cl}\{x\} \neq \operatorname{cl}\{y\}$, there exist disjoint open sets $U$ and $V$ such that $\operatorname{cl}\{x\} \subset U$ and $\operatorname{cl}\{y\} \subset V$. In [7] semi- $R_{0}$ and semi- $R_{1}$ are defined by the same manner by replacing the word open by semiopen and closure by semiclosure.

Definition 1.8. [13] $A$ space $(X, \tau)$ is a $R_{Y S^{-}}^{*}$ space if for arbitrary $x, y \in X$, $x \neq=y$ implies $D\{x\} \cap D\{y\}=\emptyset$

Definition 1.9. [19] Let $\mathrm{R}$ be the equivalence relation on space $(X, \tau)$ defined by $x R y$ iff $\operatorname{cl}\{x\}=\operatorname{cl}\{y\}$. Then the $T_{0}$-identification space of $(X, \tau)$ is $\left(X_{0}, Q\left(X_{0}\right)\right)$ where $X_{0}$ is the set of equivalence classes of $\mathrm{R}$ and $Q\left(X_{0}\right)$ is the decomposition topology on $X_{0}$.

Definition 1.10. [9] Let $\mathrm{R}$ be the equivalence relation on a space $(X, \tau)$ defined by $x R y$ iff s-cl $\{x\}=\operatorname{s-cl}\{y\}$. Then the semi- $T_{0}$-identification space of $(X, \tau)$ is $\left(X_{s}, Q\left(X_{s}\right)\right)$, where $X_{s}$ is the set of equivalence classes of $R$ and $Q\left(X_{s}\right)$ is the decomposition topology on $X_{s}$. For each $x \in X$, let $k_{x}$ be the equivalence class of $\mathrm{R}$ containing $x$.

Definition 1.11. [9] A space $(X, \tau)$ is s-essentially $T_{x}$ iff $\left(X_{s}, Q\left(X_{s}\right)\right)$ is $T_{x}$.

Definition 1.12. [14] A space $(X, \tau)$ is $E T_{x}$ (essentially $T_{x}$ ) if its $T_{0}$-identification space is $T_{x}$.

\section{Separation Properties}

Lemma 2.1. [12] $A$ space $(X, \tau)$ is a $T_{R}$ iff any non-empty closed subset of $X$ contains a closed singleton set.

Theorem 2.1. (a) If $(X, \tau)$ is $T_{R}$, then $(X, \tau(\mathcal{F}))$ is $T_{R}$.

(b) If $(X, \tau)$ is $C_{R}$, then $(X, \tau(\mathcal{F}))$ is also $C_{R}$.

Proof. (a) let $\emptyset \neq A$ be a closed set in $\tau(\mathcal{F})$, then $A=W \cup B$, where $W \in \tau^{c}$, $B \notin \mathcal{F}$. By lemma 2.1, we have that $\operatorname{cl}_{\tau}\{x\} \subset W \subset A$. Thus $\operatorname{cl}_{\tau(\mathcal{F})}\{x\} \subset A$ and 
$(X, \tau(\mathcal{F}))$ is $T_{R}$

(b) It is similar to the proof of (a)

Lemma 2.2. If $(X, \tau)$ is a space, and $(X, \tau(\mathcal{F}))$ is a filter expansion of $\tau$, then for each $x \in X$; the following are hold:

(a) $\{\hat{x}\}_{\tau(\mathcal{F})} \subset\{\hat{x}\}_{\tau}$

(b) $\left\langle x>_{\tau(\mathcal{F})} \subset<x\right\rangle_{\tau}$

(c) $D_{\tau(\mathcal{F})}\{x\} \subset D_{\tau}\{x\}$

Proof. (a) Since $\tau \subset \tau(\mathcal{F})$, then it is obvious.

(b) By (a), the proof is obvious.

(c) Since, $\operatorname{cl}_{\tau(\mathcal{F})}\{x\} \subset \operatorname{cl}_{\tau}\{x\}$, then $\operatorname{cl}_{\tau(\mathcal{F})}\{x\}-<x>_{\tau} \subset \mathrm{cl}_{\tau}\{x\}$

$$
-\langle x\rangle_{\tau} \text {. }
$$

Hence $\operatorname{cl}_{\tau(\mathcal{F})}\{x\}-\left\langle x>_{\tau(\mathcal{F})} \subset \operatorname{cl}_{\tau(\mathcal{F})}\{x\}-\left\langle x>_{\tau} \subset \operatorname{cl}_{\tau}\{x\}-\left\langle x>_{\tau}\right.\right.\right.$.

Thus $D_{\tau(\mathcal{F})}\{x\} \subset D_{\tau}\{x\}$.

Lemma 2.3 [16] A space $(X, \tau)$ is $R_{0}$ iff one of the following conditions are hold:

a) for each $x \in X, \operatorname{cl}\{x\}=<x>=\{\hat{x}\}$

b) for each $x \in X, D\{x\}=\emptyset$.

Theorem 2.2 If $(X, \tau)$ is $R_{0}$ (resp. $\left.R_{1}\right)$, then $(X, \tau(\mathcal{F}))$ is $R_{0}$ (resp. $\left.R_{1}\right)$.

Proof. By lemmas 2.2 and 2.3 .

Lemma 2.4. [1] If $(X, \tau)$ is an irresolvable space and $\tau(\mathcal{F})$ is a filter expansion of $\tau$ by afilter $\mathcal{F}$ on $X$ and $F \in S O(X, \tau)$, for every $F \in \mathcal{F}$, then $S O(X, \tau)=S O(X, \tau(\mathcal{F}))$.

Lemma 2.5. If $(X, \tau)$ is an irresolvable space and $\tau(\mathcal{F})$ is a filter expansion of $\tau$ by a filter $\mathcal{F}$ on $X$ such that $F \in S O(X, \tau)$, for every $F \in \mathcal{F}$, then for each $A \subset X, s-c l_{\tau} A=s-c l_{\tau(\mathcal{F})} A$.

Proof. Since $A \subset \mathrm{s}^{-\mathrm{cl}_{\tau(\mathcal{F})}} A$, and s-cl $\left.\mathrm{s}_{\tau} \mathcal{F}\right) A \in C(X, \tau(\mathcal{F}))=S C(X, \tau)$ [By lemma

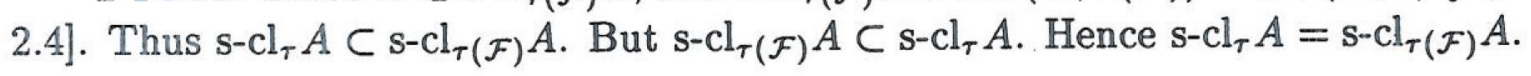

Lemma 2.6. [1] If $(X, \tau)$ is an irresolvable space and $\tau(\mathcal{F})$ is a filter expansion of $\tau$ by a filter $\mathcal{F}$ on $x$, then $S O(X, \tau) \subset S O(X, \tau(\mathcal{F}))$.

Theorem 2.3. If $(X, \tau)$ is an irresolvable and semi- $R_{i}$. Then $(X, \tau(\mathcal{F}))$ is $\operatorname{semi}-R_{i},(i \in\{0,1\})$

Proof. We will give the proof for semi- $R_{1}$, Let $x, y \in X$ such that $\operatorname{s-cl}_{\tau(\mathcal{F})}\{x\}$ $\not=\mathrm{s}^{-\mathrm{cl}_{\tau}(\mathcal{F})}\{y\}$, implies that, $\mathrm{s}-\mathrm{cl}_{\tau}\{x\} \neq \mathrm{s}-\mathrm{cl}_{\tau}\{y\}$. Since $(X, \tau)$ is semi- $R_{1}$, there exist $U, V \in S O(X, \tau)$ such that $\mathrm{s}^{-\mathrm{cl}_{\tau}}\{x\} \subset U$ and $\mathrm{s}-\mathrm{cl}_{\tau}\{y\} \subset V$, and $U \cap V=\emptyset$. But 
$S O(X, \tau) \subset S O(X, \tau(\mathcal{F}))$. Thus $(X, \tau(\mathcal{F}))$ is semi- $R_{1}$. The proof is similar for semi- $R_{0}$, spaces.

Theorem 2.4. If $(X, \tau)$ is an irresolvable space, $\tau(\mathcal{F})$ is a filter expansion of $\tau$ by a filter $\mathcal{F}$ on $X$ such that $F \in S O(X, \tau)$, for every $F \in \mathcal{F}$. Then $(X, \tau)$ is semi- $R_{i}$ iff $(X, \tau(\mathcal{F}))$ is semi- $R_{1},(i \in\{0,1\})$

Proof. From theorem 2.3, if $(X, \tau)$ is semi- $R_{1}$, then $(X, \tau(\mathcal{F}))$ is also. To prove the converse, let $x, y \in X$ such that $\mathrm{s}-\mathrm{cl}_{\tau}\{x\} \neq \mathrm{s}-\mathrm{cl}\{y\}$, implies that $\operatorname{s} \mathrm{cl}_{\tau(\mathcal{F})}\{x\} \neq$

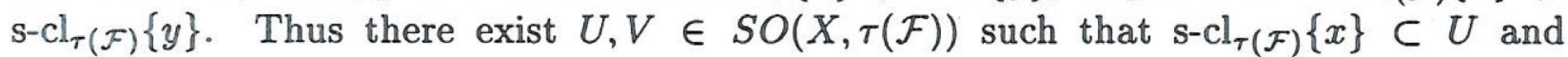
$\mathrm{s}-\mathrm{cl}_{\tau(\mathcal{F})}\{y\} \subset V$ and $U \cup V=\phi$. By lemmas 2.4 and 2.5, we arrive that $(X, \tau)$ is semi- $R_{1}$. The proof is similar for semi- $R_{0}$ spaces.

Theorem 2.5. If $(X, \tau)$ is $R_{Y S}^{*}$ then $(X, \tau(\mathcal{F}))$ is also $R_{Y S}^{*}$.

Proof. By lemma 2.2 (c)

Lemma 2.7. (a) If $A$ is nowhere dense in a space $X$, then $(X-A)$ is dense.

(b) If $A$ is dense and open subset of a space $X$, then $(X-A)$ is nowhere dense.

\section{Proof. Obvious}

Lemma 2.8. [17] Let $(X, \tau)$ be a space and $A \subset X$. Then $A \in \tau^{\alpha}$ iff $A=U-N$, such that $U \in \tau$ and $N$ is a nowhere dense set. $X$.

Remark 2.1. If $(X, \tau)$ is a submaximal space, then all dense subsets is a filter on

Theorem 2.6. If $(X, \tau)$ is a submaximal space and $\mathcal{F}$ is a filter on $X$ such that all elements are dense in $X$. Then $\tau(\mathcal{F})=\tau^{\alpha}$

Proof. Let $W \in \tau(\mathcal{F})$, then $W=U \cap A, U \in \tau$ and $A \in \mathcal{F}$. Thus $W=U-A^{c}$, and by lemma 2.7, $A^{c}$ is nowhere dense in $X$. By lemma 2.8,W $\in \tau^{\alpha}$ and $\tau(\mathcal{F}) \subset \tau^{\alpha}$. Conversely, let $W \in \tau^{\alpha}$, then $W=U-N, U \in \tau$ and $N$ is nowhere dense in $\tau$. Thus $N^{c} \in \mathcal{F}$ and $W \in \tau(\mathcal{F})$. Hence $\tau^{\alpha} \subset \tau(\mathcal{F})$.

Lemma 2.9. [2] A subset $A$ of a space $(X, \tau)$ is dense iff $A \cap U \neq \emptyset$ for each $U \in \tau-\{\emptyset\}$

Remark 2.2. It is easy to prove that lemma 2.9 still valid on replacing the word open by semi-open

Theorem 2.7 If $(X, \tau)$ is an irresolvable space and $\tau(\mathcal{F})$ is a filter expansion of $\tau$ by a filter $\mathcal{F}$ on $X$ and $F \in S O(X, \tau)$, for every $F \in \mathcal{F}$. Then $\tau$ and $\tau(\mathcal{F})$ have identical dense sets. 
Proof. By Lemma 2.4 and Remark 2.2 .

3. S-Essentially $T_{i}$-Spaces, $(i \in\{0,1\})$

Theorem 3.1 [9] Let $(X, \tau)$ be a space. Then the following are equivalent:

(a) $(X, \tau)$ is s-essentially To

(b) $X_{s}=X_{0}$

(c) If $x \in X$ such that $C_{x} \neq\{x\}$ and $O \in \tau$ such that $x \in$ cl $_{\tau} O$. Then $x \in O$,

(d) If $x \in X$ such that $C_{x} \neq\{x\}$ and $O \in S O(X, \tau)$ such that $x \in O$. Then $x \in \operatorname{int}_{\tau}(O)$.

Theorem 3.2. If $(X, \tau)$ is s-essentially $T_{0}$, then $(X, \tau(\mathcal{F}))$ is also s-essentially To.

Proof. Let $x \in X$ such that $C_{x} \neq\{x\} . \quad O \in \tau(\mathcal{F})$ and $x \in \operatorname{cl}_{\tau(\mathcal{F})} O$. Then $O=U \cap F, U \in \tau$ and $F \in \mathcal{F}$ and $\operatorname{cl}_{\tau(\mathcal{F})} O=\operatorname{cl}_{\tau(\mathcal{F})}(U \cap F) \subset \mathrm{cl}_{\mathcal{\tau}(\mathcal{F})} U \cap \mathrm{cl}_{\tau(\mathcal{F})} F \subset$ $\operatorname{cl}_{\tau(\mathcal{F})} U \subset \mathrm{cl}_{\tau} U$. Hence $x \in \operatorname{cl}_{\tau} U$. But $(X, \tau)$ is s-essentially $T_{0}$, implies that $x \in U$. There are two cases:

(a) If $x \in F$, then $x \in U \cap F=O$. By Theorem $3.1(X, \tau(\mathcal{F}))$ is s-essentially To.

(b) If $x \notin F$, then $x \notin U \cap F=O$. Let $y \in C_{x}$ such that $y \neq x$ implies that $\operatorname{cl}_{\tau(\mathcal{F})}\{y\}=$ $\mathrm{cl}_{\tau(\mathcal{F})}\{x\} \subset \mathrm{cl}_{\tau(\mathcal{F})} O$, since $x \notin O$, then $x \in(X-O) \in[\tau(\mathcal{F})]^{c}$. Hence $\operatorname{cl}_{\tau(\mathcal{F})}\{x\} \subset X-O$, which implies that $y \notin \mathrm{cl}_{\tau(\mathcal{F})}\{x\}$. This is a contractiction .

Theorem 3.3. [9] If $(X, \tau)$ is a noncompact space and $p \notin X$ such that $X^{*}=X \cup\{p\}$. Let $\tau^{*}$ be the one-point compactification topology on $X^{*}$, and let $i:(X, \tau) \rightarrow\left(X^{*}, \tau^{*}\right)$ be the identity mapping. Then $(X, \tau)$ is s-essentially To iff $\left(X^{*}, \tau^{*}\right)$ is s-essentially $T_{0}$.

Theorem 3.4 If $(X, \tau)$ is a noncompact space and $p \notin X$ such that $X^{*}=$ $X \cup\{p\}$, and $\tau^{*}$ is the one -point compactification topology on $X^{*}$, and let $i$ : $(X, \tau) \rightarrow\left(X^{*}, \tau^{*}\right)$ be the identity mapping and $(X, \tau)$ is s-essentially $T_{0}$. Then $(X, \tau(\mathcal{F}))$ and $\left(X^{*}, \tau^{*}(\mathcal{F})\right)$ are s-essentially $T_{0}$.

Proof. By theorems 3.2 and 3.3

Definition 3.1. [8] If $\mathrm{f}$ is a continuous function from a space $(X, \tau)$ onto $(Y, S)$, then the function $f^{*}:\left(X_{0}, Q(\tau)\right) \rightarrow\left(Y_{0}, Q(S)\right)$ defined by $f^{*}\left(C_{x}\right)=C_{f(x)}$ is the induced map from $\left(X_{0}, Q(\tau)\right)$ onto $\left(Y_{0}, Q(S)\right)$ determined by $f$.

Theorem 3.5. [8] If $f$ is a continuous function from $(X, \tau)$ onto $(Y, S)$, then the relation $f^{*}=\left\{\left(C_{x}, C_{f(x)}\right) / C_{x} \in X_{0}\right\}$ is a continuous function from $\left(X_{0}, Q(\tau)\right)$ onto $\left(Y_{0}, Q(S)\right)$.

Theorem 3.6. [8] Let $f$ be a continuous function from $(X, \tau)$ onto $(Y, S)$. If 
$f$ is open (closed), then $f^{*}$ is open (closed).

Theorem 3.7. Let $f:(X, \tau) \rightarrow(X, \tau(\mathcal{F}))$ be a homeomorphic mapping and $(X, \tau)$ be a compact space, then:

(i) $f^{*}:\left(X_{0}, Q(\tau)\right) \rightarrow\left(X_{0}, Q(\tau(\mathcal{F}))\right.$ is also a homeomorphic mapping.

(ii) $(X, \tau(\mathcal{F})),\left(X_{0}, Q(\tau)\right),\left(X_{0}, Q(\tau(\mathcal{F}))\right.$ are also compact spaces.

Proof. By using theorems 3.5 and 3.6.

By Theorem 3.7, we give the following diagram (where $P_{q}$ is projection mapping of $X$ onto $X_{0}[19]$.

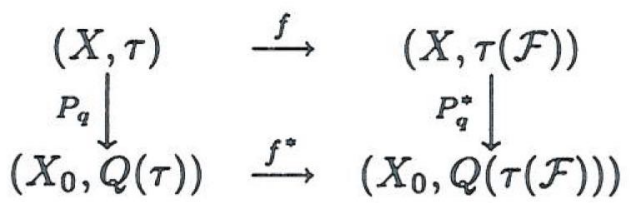

Theorem 3.8. [16] A space $(X, \tau)$ is $E T_{Y S}$ iff for $x, y \in X,\langle x\rangle \neq\langle y\rangle$ implies $D\{x\} \cap D\{y\}=\emptyset$

Theorem 3.9. If $(X, \tau)$ is $\operatorname{ET}_{Y S}$, then $(X, \tau(\mathcal{F}))$ is also ETYS.

Proof. By Theorem 3.8, and Lemma. 2.2

Theorem 3.10. If $(X, \tau)$ is $E T_{1}$ (or $\left.E T_{D}\right)$, then $(X, \tau(\mathcal{F}))$ is $E T_{1}$ (or $\left.E T_{D}\right)$.

Proof. By Lemma 2.2., the proof is obvious.

\section{References}

[1] M. E. Abd EL-Monsef, E. F. Lashin, Filter extension of topologies, Proc. 3rd Conf. Math. Stat. O.R. and Appl. Bull. Fac. Sci. Alex. Univ. (1988).

[2] N. Bourbaki, Elements of mathematics, General Topology, Part I (Herman, Paris, Addison -Wesley, Reading, Mass. 1966).

[3] D. E. Cameron, "Aclass of maximal topologies", Pac. J. of Math. 70 (1977), 101-104.

[4] S. G. Crossley, S.K. Hildebrand, "Semi-closure", Texas J. Sci. 22 (1971), 99-112.

[5] A. Davis, "Indexed systems of neighborhoods for general topological spaces", Amer. Math. Monthly, 68 (1961), 886-893.

[6] C. H. Dorsett, " $R_{0}$ and $R_{1}$ topological spaces", Math. Vesnik, 2 (15) (30), No.2 (1978), 117-122

[7] C. H. Dorsett, "Semi- $T_{2}$, semi- $R_{1}$ and semi- $R_{0}$ topological spaces", Ann. Soc. Sci. Bruxelles, Ser. I, 92 (1978), 143-159.

[8] C. H. Dorsett, "T $T_{0}$-identification spaces and $R_{1}$ spaces", Kyungpook Math. J. Volume 18, N. 2 (1978).

[9] C. H. Dorsett, "Semi- $T_{0}$-identification spaces and $s$-essentially $T_{0}$-spaces", Prre Math. Manuscript 3 (1984), 23-32.

[10] C. H. Dorsett, Semi- $T_{0}$-identification spaces and $s$-essentially $T_{1}$-spaces", Eleutheria Math. J. Sem. P. Zervas, 3 (1988), 4-16.

[11] E. Hewitt, "A problem of set theoretic topology", Duke. Math. J. 10 (1943), 309-333. 
[12] G. Josep, " $T_{D^{2}}$ spaces and other axioms weaker than $T_{0}$ ", Act. Colloquim on topology (eger, Hungary, 1983).

[13] G. Josep, "Axioms weaker than $R_{0}$ ", Mat. Vesnik 36 (1984), 195-205.

[1.4] G. Josep, "Essentially $T_{D}$ and essentially $T_{U D}$ spaces", Bull. Math. Soc. Sci. Math. (Roumanie) 32 (80) N. 3 (1988).

[15] N. Levine, "Semiopen sets and semi continuity in topological spaces", Amer. Math. Monthly 70 (1963), 36-41.

[16] D.A. Maria and G. Josep, "On the separation oxiom $R_{T}$ ", Kyungpook Math. J. Volume 26 N. 1 (1986), 67-72.

[17] O. Njasted, "On some classes of nearly open sets", Pac. J. of Math. 15 (1965), 961-970.

[18] M. W. Warner, "Some separation axioms weaker than To. Bull. Malaysian Math. Soc. 6 (1975), No. 3, 28-34.

[19] S. Willard, General topology, Addison Wesley Publishing Co., 1970.

Department of Mathematics, Faculty of Science, Tanta University, Tanta, Egypt. 